\title{
Síndrome alcoólica fetal - revisão sistematizada
}

\author{
Fetal alcohol syndrome - sistematic review \\ Síndrome alcohólico fetal - revisión sistemática \\ Rogério A. Santana, Leonardo F. J. L. Almeida, Denise L. M. Monteiro*
}

\section{Resumo}

A ingestão de álcool (etanol) pela mãe durante a gravidez pode resultar em variedade de consequências relacionadas ao neurodesenvolvimento, incluindo retardo mental e deficiências em capacidade cognitiva, atenção, função executiva, controle motor e comportamento. O conjunto dessas manifestações é conhecido como Síndrome Alcóolica Fetal (SAF). O objetivo deste estudo é identificar os possíveis efeitos do uso do etanol durante a gravidez sobre o embrião humano. Foi realizada ampla pesquisa na literatura médica procurando identificar e extrair informações da literatura nacional e internacional por meio das seguintes bases de dados: Medline (por meio do PubMed), SciELO, LILACS e Cochrane. As principais complicações dos fetos, cujas mães fizeram uso do álcool na gravidez são: baixo peso ao nascer (BPN), crescimento intrauterino restrito (CIUR), prematuridade, retardo no neurodesenvolvimento e microcefalia. O uso de álcool entre as mulheres em idade fértil é causa evitável de anomalias congênitas e deficiências de desenvolvimento. Para prevenir a SAF é aconselhável evitar o uso do álcool na gestação, visto que ainda é difícil definir a dose mínima que irá afetar o desenvolvimento do embrião.

Descritores: Síndrome alcoólica fetal; Alcoolismo; Gestação.

\begin{abstract}
The consumption of alcohol (ethanol) by the mother during the pregnancy can result in a range of consequences related to the neurodevelopment, including mental delay and deficiencies in the cognitive capacity, attention, executive function, motor control and behavior. The assembly of those manifestations is known like Fetal Alcoholic Syndrom (SAF). The objective of this study is going to identify the possible effects of the use of the ethanol during the pregnancy about the human embryonary. It was carried out broad research in the medical literature finding identify and extract information of the international and national literature by means of the following databases: Medline (by means of the PubMed), SciELO, LILACS and Cochrane. The main complications of the fetuses, whose mothers did use of the alcohol in the pregnancy are: low birth weight, intrauterine growth restriction, prematurity, delay in the neurodevelopment and microcefalia.The use of alcohol between the women in fertile age is avoidable cause of congenital anomalies and deficiencies of development. To prevent SAF is recommended to avoid alcohol in the gestation, due to the fact that still difficult to defined the minimum dose that is going to affect the development of the embryo.
\end{abstract}

Keywords: Fetal alcohol syndrome; Alcoholism; Pregnancy.

\section{Resumen}

La ingestión de alcohol (etanol) por la madre durante el embarazo puede resultar en una variedad de consecuencias relacionadas al neurodesarrollo, incluyendo retardo mental y deficiencias en capacidad cognitiva, atención, función en la ejecución, control motor y comportamiento. El conjunto de esas manifestaciones es conocido como síndrome alcohólico fetal (SAF). El objetivo de este estudio es identificar los posibles efectos del uso de etanol durante el embarazo sobre el embrión humano. Se realizó una amplia investigación en la literatura médica buscando identificar y extraer informaciones de la literatura nacional e internacional por medio de las siguientes bases de datos: Medline (por medio del PubMed) SciELO, LILACS y Cochrane. Las principales complicaciones de los fetos, cuyas madres hicieron uso de alcohol en el embarazo son: bajo peso al nacer (BPN), crecimiento 
intrauterino restricto (CIUR), prematuridad, retraso en el neurodesarrollo y microencefalia. El uso de alcohol entre las mujeres en edad fértil es una factor evitable para anomalías congénitas y deficiencias de desarrollo. Para prevenir la SAF es aconsejable evitar el uso del alcohol en la gestación, ya que todavía es difícil definir la dosis mínima que afectará el desarrollo del embrión.

Palabras clave: síndrome alcohólico fetal; alcoholismo; gestación

\section{Introdução}

O relatório da Organização Mundial da Saúde (OMS) de 2002 apontou o alcoolismo como sendo o principal problema de saúde na América Latina. No Brasil, a relação é de três homens consumidores de álcool para cada mulher; porém, esse número cresce entre as mulheres severa e precocemente, inclusive entre gestantes.'(D)

A ingestão de álcool (etanol) durante a gravidez pode resultar em várias consequências relacionadas ao neurodesenvolvimento, incluindo retardo mental e deficiências em capacidade cognitiva, atenção, função executiva, controle motor e comportamento, conhecida como Síndrome Alcóolica Fetal (SAF). ${ }^{2}$ (A) Embora a maioria das mulheres reduza ou interrompa o consumo de álcool ao descobrir a gravidez, algumas delas continuam consumindo na mesma ou até em maiores quantidades. $^{3}$ (C) Estima-se que mais de 1 em cada 100 nascidos vivos apresentem manifestações relacionadas ao consumo de álcool na gestação. ${ }^{2}(\mathrm{~A})$ O Centro de Controle e Prevenção de Doenças (CDC) identificou que 7,6\% das mulheres grávidas faziam uso de álcool e 1,4\% admitiram fazer uso abusivo durante a gestação. ${ }^{4}(\mathrm{~B})$

Ethen e colaboradores (2009) observaram uso eventual de álcool em 30,3\% das gestantes e ingestão de álcool durante toda a gestação em
$2,7 \%$. Beber antes da gravidez foi fator preditor $(\mathrm{OR}=8,5 ; \mathrm{IC} 95 \%=6,7-10,9)$. O maior uso de álcool durante a gestação foi associado à idade entre 35 e 44 anos, fumar e não ter planejado a gravidez. ${ }^{5}$ (B) Os fatores de risco associados ao consumo de álcool durante a gestação incluem: idade maior que 25 anos, baixo nível socioeconômico, tabagismo, ser solteira, desempregada, usuária de drogas ilícitas, história de abuso sexual ou físico ou negligência, história de encarceramento, ter parceiro ou membro da família que bebe muito e apresentar estresse psicológico ou distúrbio da saúde mental. ${ }^{6,7}$ (D)

Neste estudo, discutiremos os possíveis efeitos do uso do etanol durante a gravidez sobre o embrião humano e as possíveis repercussões fetais.

\section{Métodos}

Foi realizada uma revisão sistematizada após ampla pesquisa na literatura médica procurando identificar e extrair informações das seguintes bases de dados: Medline (por meio do PubMed), Scielo, LILACS e Cochrane. Para identificação de publicações no PubMed, foi utilizada a estratégia de busca: "Pregnancy"[Mesh]) AND "Alcoholism"[Mesh]) AND "Risk"[Mesh], tendo sido encontrados 97 artigos. Os descritores da

\section{Quadro 1. Critérios para o diagnóstico de DMG da International Association of Diabetes and Pregnancy Study} Groups - 2010.

\begin{tabular}{|c|c|}
\hline Critérios de inclusão & Critérios de exclusão \\
\hline $\begin{array}{l}\text { 1. Estudos sobre a relação entre uso de álcool na } \\
\text { gestação e a síndrome alcoólica fetal. } \\
\text { 2. Estudos que abordam os efeitos do álcool sobre o } \\
\text { desenvolvimento fetal. } \\
\text { 3. Estudos sobre consequências fetais em gestações } \\
\text { nas quais as mães fizeram uso do álcool. } \\
\text { 4. Estudos que falam exclusivamente do uso do álcool } \\
\text { 5. Acarretando repercussões fetais. } \\
\text { 6. Estudos publicados entre } 2003 \text { e } 2013 \text {. }\end{array}$ & $\begin{array}{l}\text { 1. Artigos baseados em estudos com animais. } \\
\text { 2. Textos que não falam da relação entre alcoolismo na } \\
\text { gestação e a síndrome alcoólica fetal. } \\
\text { 3. Artigos que abordam as consequências maternas do } \\
\text { uso do álcool na gestação. } \\
\text { 4. Artigos que incluem outras drogas, além do álcool } \\
\text { (mesmo abordando consequências fetais). } \\
\text { 5. Estudos sobre o prognóstico de bebês com SAF após } \\
\text { 6. Antervenção. }\end{array}$ \\
\hline
\end{tabular}




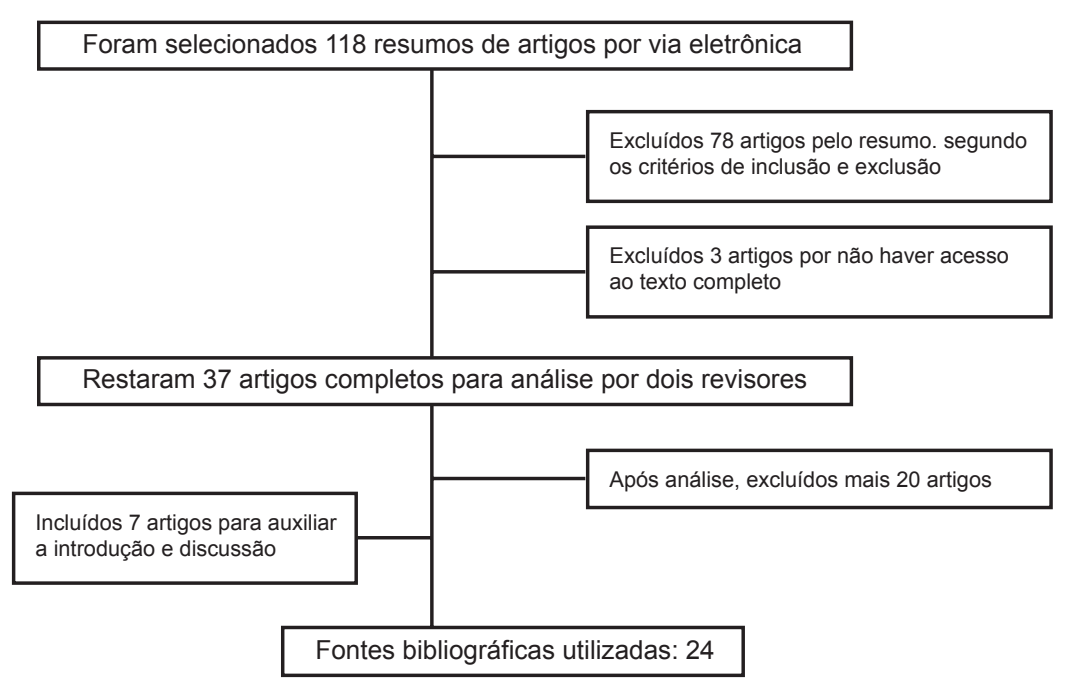

Figura 1. Fluxograma de busca de referências.

\section{Quadro 2.}

\begin{tabular}{|c|l|}
\hline \multicolumn{2}{|c|}{ Grau de recomendação e força de evidência } \\
\hline A & $\begin{array}{l}\text { Estudos experimentais ou observacionais de melhor consistência (metanálises ou ensaios clínicos } \\
\text { randomizados). }\end{array}$ \\
\hline B & $\begin{array}{l}\text { Estudos experimentais ou observacionais de menos consistência (outros ensaios clínicos não randomizados } \\
\text { ou estudos observacionais ou estudos caso-controle). }\end{array}$ \\
\hline C & Relatos ou séries de casos (estudos não controlados). \\
\hline D & Opinião desprovida de avaliação crítica, baseada em consensos, estudos fisiológicos ou modelos animais. \\
\hline
\end{tabular}

pesquisa usados nas outras bases de dados foram: síndrome alcóolica fetal, alcoolismo, gravidez, etanol, complicações na gravidez, feto, pregnancy e alcoholism, sendo encontrados mais 21 artigos. Foram excluídos 78 artigos pelo resumo, obedecendo-se aos critérios de inclusão e exclusão pré-estabelecidos (Quadro 1). Três artigos foram excluídos por não ter sido possível o acesso aos textos completos. Após a análise dos 37 artigos por dois revisores, foram selecionados 17 artigos. Ainda foram incluídos sete artigos de revisão para auxiliar na confecção da introdução e discussão, totalizando 24 referências.

O detalhamento da forma de seleção dos artigos utilizados na confecção deste estudo se encontra no fluxograma (Figura 1).

Para a classificação dos trabalhos citados na revisão, utilizou-se a classificação proposta pela Associação Médica Brasileira (Quadro 2).

\section{Resultados}

Os estudos que abordam as principais complicações fetais de gestações de usuárias de álcool estão detalhados na tabela 2 .

De acordo com os resultados encontrados na literatura e apresentados nessa tabela, as principais complicações da SAF são: prematuridade, CIUR, BPN e microcefalia.

\section{Discussão}

Vários medicamentos e produtos químicos são conhecidos por serem teratogênicos para o embrião humano quando administrados durante a gravidez, especialmente durante o período de organogênese. Estes insultos teratogênicos que ocorrem durante a vida embrionária podem estar presentes imediatamente após o nascimento, na 
Tabela 2. Principais complicações fetais da Síndrome Alcoólica Fetal (SAF)

\begin{tabular}{|c|c|c|c|}
\hline Estudo/Ano & Desenho de estudo & Amostra & Resultados \\
\hline $\begin{array}{l}\text { Hutson Jr. et al } \\
(2012)^{8}\end{array}$ & Caso controle (B) & 23 casos/24 controles & $\begin{array}{l}\text { Uso de álcool na gravidez prejudica o trans- } \\
\text { porte de folato para o feto devido à alteração } \\
\text { na expressão de proteínas de ligação e trans- } \\
\text { porte, causando defeito na formação tubo } \\
\text { neural e anomalias cardíacas }\end{array}$ \\
\hline $\begin{array}{l}\text { Feldman HS } \\
\text { et al }(2012)^{9}\end{array}$ & Coorte (A) & 992 & $\begin{array}{l}\text { Uso com } 6-12 \text { semanas gestação: microcefalia } \\
\text { e BPN. } N^{0} \text { de bebidas (RR=1,25). Redução do } \\
\text { comprimento em qualquer trimestre }\end{array}$ \\
\hline $\begin{array}{l}\text { Silva I et al } \\
(2011)^{10}\end{array}$ & Corte transversal (C) & 957 & Prematuridade e BPN $(p<0,03)$ \\
\hline $\begin{array}{l}\text { Manzardo AM } \\
\text { et al }(2011)^{11}\end{array}$ & Coorte (B) & 448 & $\begin{array}{l}\text { Efeitos gênero específicos: retardo no neu- } \\
\text { rodesenvolvimento, crescimento e prematuri- } \\
\text { dade (maior no sexo masculino) }\end{array}$ \\
\hline $\begin{array}{l}\text { Solonskii AV } \\
\text { et al (2008) }\end{array}$ & Caso controle (B) & 23 casos/30 controles & $\begin{array}{l}\text { O álcool leva à hipóxia fetal causando CIUR } \\
\text { e aumento do número de vasos cerebrais, } \\
\text { porém com lúmen reduzido. }\end{array}$ \\
\hline $\begin{array}{l}\text { Garcia-Algar O } \\
\text { et al (2008) } 13\end{array}$ & Coorte (B) & 353 & $\begin{array}{l}\text { Álcool foi associado a menor número de nas- } \\
\text { cimentos pré-termo e permite o aumento da } \\
\text { composição de gordura corporal do feto }\end{array}$ \\
\hline
\end{tabular}

BPN - baixo peso ao nascer; CIUR - crescimento intrauterino restrito

infância ou até mais tarde na vida adulta, especialmente se o dano envolve o Sistema Nervoso Central (SNC). ${ }^{14}$ (B) Além disso, muitos dos efeitos teratogênicos no SNC podem ocorrer no segundo e no terceiro trimestres de gravidez, quando a maioria dos outros órgãos já passou da fase de organogênese ativa (o córtex cerebral, por exemplo, continua a se desenvolver ativamente durante toda a gestação, principalmente ao formar as diferentes camadas corticais, no crescimento neuronal e brotação de formação, sinapse e mielinização). ${ }^{14,15}$ (B) Assim, tal efeito tardio não será necessariamente manifestado por alterações morfológicas no SNC, mas sim por mudanças mais sutis na capacidade intelectual, capacidade de aprendizagem de atenção e comportamento.

Quanto à quantidade de álcool ingerida necessária para afetar o desenvolvimento fetal, parece haver três categorias de exposição pré-natal ao etanol relacionada com a quantidade de álcool ingerida. O grau elevado de exposição (mais de 48-60 g de etanol/dia) pode causar a síndrome alcoólica fetal; exposição moderadamente alta ao álcool (entre 24 e 48 g etanol/dia), o que pode resultar na maior parte em "os efeitos do álcool" (as diferenças entre estas categorias não são nítidas); e exposições ocasionais ao álcool (no total mais de 90 g etanol/bebida). ${ }^{16,17}$ (B)

Quanto às características da síndrome alcoólica fetal, as principais incluem: atraso no crescimento pré e pós-natal ${ }^{9,11}(\mathrm{~A}, \mathrm{~B})$, baixa estatura ${ }^{9}$ (A), atraso no desenvolvimento" ${ }^{11}(B)$, microcefalia $^{9}$ (A), BPN, ${ }^{8,9}(\mathrm{~B}, \mathrm{~A})$ dificuldade na realização de movimentos finos e dismorfismo facial. Além disso, pode haver fenda palatina, anomalias articulares e cardíacas e vincos alterados palmares. $\mathrm{O}$ dismorfismo facial descrito acima tende a melhorar com o avanço da idade dos indivíduos afetados. Hutson e colaboradores $(2012)^{8}$ mostraram que o uso de álcool na gravidez prejudica o transporte de folato para o feto devido à alteração na expressão de proteínas de ligação e transporte, acarretando defeito na formação do tubo neural e anomalias cardíacas. O álcool pode levar à hipóxia fetal causando CIUR e aumento do número de vasos cerebrais, porém com lúmen reduzido. ${ }^{12}$ (B) Além disso, a prematuridade é considerada importante consequência da Síndrome Alcoólica Fetal $^{8,11}$ (B). No entanto, em um estudo de coorte (B), o álcool foi associado a menor número de nascimentos pré-termo, mas como parcela da população estudada consumia outras drogas como a maconha e cocaína, este fato pode ter influenciado esse resultado. 
O diagnóstico de SAF é baseado em alguns critérios como: deficiência de crescimento, fenótipo facial e danos no sistema nervoso central, associados à história de exposição ao álcool na gestação.

Em um estudo de coorte (B), incluindo 24.679 gestações, foi observado risco aumentado de aborto espontâneo no primeiro trimestre (de 7 a 11 semanas completas de gestação) para as mulheres que consomem $\geq 5$ drinques / semana. No entanto, não foi encontrada associação entre o consumo de álcool e aborto espontâneo durante o segundo trimestre. ${ }^{18}$ (B)

Os principais mecanismos para explicar os efeitos teratogênicos do álcool sobre o embrião em desenvolvimento incluem: (1) aumento do estresse oxidativo (formação de radicais livres); (2) distúrbio no metabolismo da glicose, proteínas, lipídios e DNA; (3) neurogênese diminuída e aumento da apoptose celular, em particular de células da crista neural. ${ }^{19-22}$ (D)

A principal limitação deste estudo foi a heterogeneidade dos estudos que avaliaram as complicações fetais, não apresentando resultados idênticos a respeito dos sinais e sintomas da SAF, exceto em relação ao crescimento. Também não foi possível definir a dose mínima ideal de álcool para consumo durante a gravidez. O ideal, para prevenir a SAF, é suspender o consumo de álcool durante a gestação, visto que ainda não existe um consenso quanto à dose mínima necessária para afetar o desenvolvimento do embrião. ${ }^{23}$ Caso a mãe tenha feito uso de álcool durante a gestação e supondo-se que o estresse oxidativo é uma das principais vias de danos induzidos pelo etanol, uma medida que pode ser tomada é a suplementação antioxidante, num esforço para atenuar este dano. Os antioxidantes, como a vitamina $\mathrm{C}$, vitamina E, ácido fólico, betacaroteno e os flavonoides podem ser complementados pela ingesta de alimentos. ${ }^{24}$ (D)

\section{Conclusão}

A ingestão de álcool durante a gestação pode ter efeitos deletérios sobre o SNC, crescimento e desenvolvimento do feto em graus variados, dependendo da duração da exposição e da época da gestação. De acordo com os resultados encontrados, as principais complicações fetais em gestantes que fizeram uso do álcool são: baixo peso ao nascer, CIUR, prematuridade, retardo no neurodesenvolvimento e microcefalia.

Assim sendo, o ideal é suspender o consumo de álcool durante a gestação, já que não existe consenso sobre qual é a dose mínima capaz de afetar o desenvolvimento fetal. Tendo em vista os resultados deste estudo, medidas educativas devem ser aplicadas para mulheres em idade fértil usuárias de bebidas alcoólicas visando prevenir a instalação da Síndrome Alcoólica Fetal.

\section{Referências}

1. Silva EAF. Alcoolismo e a mulher. Bezerra Informa. 2003;11:4-5

2. Boehm SL 2 $2^{\text {nd }}$, Moore EM, Walsh CD, Gross CD, Cavelli AM, Gigante E, et al Using drinking in the dark to model prenatal binge-like exposure to ethanol in C57BL/6J mice. Dev Psychobiol. 2008;50(6):566-78.

3. Floyd RL, Weber MK, Denny C, O'Connor MJ Prevention of fetal alcohol spectrum disorders. Dev Disabil Res Rev. 2009;15(3):193-9.

4. MMWR. Alcohol Use and Binge Drinking Among Women of Childbearing Age - United States, 2006-2010. Disponível em: http://www.cdc.gov/ mmwr/preview/mmwrhtml/mm6128 a4.htm?s_ cid=mm6128a4_e. Acesso em: 19 Jul 2013.

5. Ethen MK, Ramadhani TA, Scheuerle AE, Canfield MA, Wyszynski DF, Druschel CM, et al. Alcohol consumption by women before and during pregnancy. Matern Child Health J. 2009; 13(2):274-85. http://dx.doi: 10.1007/s10995-008-0328-2.

6. Floyd RL, O’Connor MJ, Sokol RJ, Bertrand J, Cordero JF. Recognition and prevention of fetal alcohol syndrome. Obstet Gynecol. 2005;106(5 Pt 1):1059-64

7. Fetal Alcohol Syndrome: Guidelines for referral and Diagnosis. National Center on Birth Defects and Developmental Disabilities Centers for Disease Control and Prevention Department of Health and Humam Services. July 2004. Available at www.cdc. gov/ncbddd/fas/default.htm. Acesso em 10 Jan 2013.

8. Hutson JR, Stade B, Lehotay DC, Collier CP, Kapur BM. Folic acid transport to the human fetus is decreased in pregnancies with chronic alcohol exposure. PLoS One. 2012;7(5):e38057. http://dx.doi: 10.1371/journal.pone.0038057

9. Feldman HS, Jones KL, Lindsay S, Slymen D, KlonoffCohen H, Kao K, et al. Prenatal alcohol exposure patterns and alcohol-related birth defects and growth deficiencies: a prospective study. Alcohol Clin Exp Res. 2012;36(4):670-6. http://dx.doi. 
org/10.1111/j.1530-0277.2011.01664.x

10. Silva I, Quevedo L de A, Silva RA, Oliveira SS, Pinheiro RT. Association between alcohol abuse during pregnancy and birth weight. Rev Saude Publica. 2011;45(5):864-9.

11. Manzardo AM, Madarasz WV, Penick EC, Knop J, Mortensen EL, Sorensen HJ, et al. Effects of premature birth on the risk for alcoholism appear to be greater in males than females. J Stud Alcohol Drugs. 2011 ;72(3):390-8.

12. Solonskii AV, Logvinov SV, Kutepova NA.

Development of brain vessels in human embryos and fetuses in conditions of prenatal exposure to alcohol. Neurosci Behav Physiol. 2008;38(4):373-6.

13. Garcia-Algar O, Kulaga V, Gareri J, Koren G, Vall O, Zuccaro P, et al. Alarming prevalence of fetal alcohol exposure in a Mediterranean city. Ther Drug Monit. 2008;30(2):249-54.

14. Ornoy A. The impact of intrauterine exposure versus postnatal environment in neurodevelopmental toxicity: long-term neurobehavioral studies in children at risk for developmental disorders. Toxicol Lett.2003;140-141:171-81.

15. Ornoy A. The effects of alcohol and illicit drugs on the human embryo and fetus. Isr J Psychiatry Relat Sci. 2002;39:120-32.

16. Kesmodel U, Wisborg K, Olsen SF, Henriksen TB, Secher NJ. Moderate alcohol intake in pregnancy and the risk of spontaneous abortion. Alcohol. 2002;37(1):87-92.

17. Martinez-Frias ML, Bermejo E, Rodriguez-Pinilla E, Frias JL. Risk for congenital anomalies associated with different sporadic and daily doses of alcohol consumption during pregnancy: a case-control study. Birth Defects Res A Clin Mol Teratol. 2004;70(4):194-200.

18. Kesmodel U, Wisborg K, Olsen SF, Henriksen TB, Secher NJ. Moderate alcohol intake during pregnancy and the risk of stillbirth and death in the first year of life. Am J Epidemiol. 2002;155(4):305.

19. Ornoy A. Embryonic oxidative stress as a mechanism of teratogenesis with special emphasis on diabetic embryopathy. Reprod Toxicol. 2007;24:31-41.
20. Cartwright MM, Smith SM. Increased cell death and reduced neural crest cell numbers in ethanolexposed embryos: partial basis for the fetal alcohol syndrome phenotype. Alcohol Clin Exp Res. 1995;19:378-86.

21. Heaton MB, Paiva M, Mayer J, Miller R. Ethanolmediated generation of reactive oxygen species in developing rat cerebellum. Neurosci Lett. 2002;334:83-6.

22. Kay HH, Tsoi S, Grindle K, Magness RR. Markers of oxidative stress in placental villi exposed to ethanol. J Soc Gynecol Investig. 2006;13:118-21.

23. Ornoy A, Ergaz Z. Alcohol abuse in pregnant women: effects on the fetus and newborn, mode of action and maternal treatment. Int J Environ Res Public Health. 2010;7(2):364-79. http://dx.doi.org/10.3390/ ijerph7020364.

24. Cohen-Kerem R, Koren G. Antioxidants and fetal protection against ethanol teratogenicity. I. Review of the experimental data and implications to humans. Neurotoxicol Teratol. 2003;25:1-9.

\section{Rogério A. Santana}

Faculdade de Medicina. Centro Universitário Serra dos Órgãos (UNIFESO). Teresópolis, RJ, Brasil.

\section{Leonardo F. J. L. Almeida}

Faculdade de Medicina. Centro Universitário Serra dos Órgãos (UNIFESO). Teresópolis. RJ, Brasil.

\section{Denise L. M. Monteiro}

Disciplina de Obstetrícia. Departamento de Ginecologia e Obstetrícia. Centro Universitário Serra dos Órgãos (UNIFESO). Teresópolis, RJ, Brasil.

Disciplina de Obstetrícia. Departamento de Ginecologia e Obstetrícia. Faculdade de Ciências Médicas da Universidade do Estado do Rio de Janeiro. Rio de Janeiro, RJ, Brasil. 